\title{
Currents in Line Conductors due to an Electron Avalanche
}

\author{
Debasish Nath ${ }^{1 *}$, Udaya Kumar ${ }^{1}$ \\ ${ }^{1}$ Department of Electrical Engineering, Indian Institute of Science, Bangalore, India \\ *debasishn@iisc.ac.in
}

\begin{abstract}
Transfer of bulk electric power required in the modern world can be realized only through EHV and UHV transmission lines. The scenario is dominated by overhead lines in which electromagnetic noise generated by corona is an important concern. Corona induced currents propagate along line conductors producing electromagnetic noise, which is essential to be quantified. In literature, large amount of work is based on experimental investigations which considers only limited frequency ranges and is not applicable to new line configurations. A set of semi-analytical methods have also been proposed, which employ the transmission line model for analysis. However, quasi-TEM mode of propagation inherently assumed by them has been questioned. Moreover, the corona current is modeled as shunt source without relating to the mechanism of induction due to corona. The present work aims to investigate the basic mechanism of current induction using an isolated avalanche developing under space charge modulated background field. The corresponding induced currents are quantified and the structure of the electric and magnetic fields is extracted. The basic issue with the long transmission line modelling is amply demonstrated. Even though single avalanche, being the basic process building corona is considered, general inferences can be drawn regarding corona on lines.
\end{abstract}

\section{Introduction}

In the transmission of electric power, fast electromagnetic transients are often experienced in power systems. Corona on overhead line conductors is one of the important phenomena which can produce such transients. Corona induces in the phase conductors and ground wire, currents, which are pulsed in nature and possess very fast rise time (in tens of ns), followed by a relatively slow tail time (few hundreds of ns) [1]. The pulsed currents propagate along the lines producing Electromagnetic noise (EM noise) in the neighbourhood and may lead to Electromagnetic Interference (EMI). In the present times when Electromagnetic Compatibility (EMC) is of utmost importance, the noise produced by power lines under corona has become very significant.

A number of methods have been developed to quantify the EM noise due to corona on transmission lines which can be broadly classified into two categories [1]: 1) Empirical or experimental methods and 2) Semi- empirical or semianalytical methods.

The salient features of the empirical methods are:

a). Basic assumption employed is; if the surface gradient and conditions in the vicinity of the conductor are duplicated, then the corona per unit length is also replicated [1].

b). By duplicating the surface gradient on a reference or test lines (usually single phase, 2-3 spans in length), measurements are carried out [1].

c). The data obtained is utilized to arrive at an empirical relation for the prediction of the electromagnetic noise from actual three phase lines [1].

d). Due to variations in the line configurations and geometry between reference or test lines and the actual power lines, weather conditions, correction factors are employed in arriving at the empirical relations [1].
The limitations can be summarized as:

a). It is not always possible to replicate the conditions in the vicinity of the line conductors

c). The correction factors can only be applied to incorporate a limited range of variations. This poses a major limitation as higher and higher voltages are considered along with newer line configurations.

d). For new line configurations, the measured data is not readily available and the prevailing correction factors may not be applicable.

e). Moreover, measured data from reference lines collected over a number of decades cater to only a limited frequency range, typically the AM Radio frequency range (535- 1605 $\mathrm{kHz}$ ). In the present times where EMI and EMC are of major concern, such a limited frequency range is clearly inadequate.

The semi-analytical approach attempts to address some of the limitations of empirical methods. In these methods, the corona current is assumed to be injected into the transmission line conductors (in the form of shunt current sources) and evaluation of the field is carried out with the help of the transmission line model. However, for the propagation of pulsed corona current, it is believed that the transmission line may behave like a radiating antenna [1]. As a result, the use of transmission line model has been questioned in the literature $[1,2]$.

In order to answer the above questions, the formulation and solution of the problem needs to be carried out based on Maxwell's equations. Therefore, before the EM noise from transmission line due to corona can be quantified, aspects related to the methodology needs to be studied. Some of these are taken up in the present work.

The present work is therefore not aimed at quantifying the noise produced by power lines under corona. It is modelling the physics of the problem following a complete field-based 
(Maxwell's equation) approach which is the objective at present. In order to adopt a complete field-based approach, the following would be necessary:

a). Computation of the induced current by the full wave solution of Maxwell's equation:

In literature, since transmission line model with injected corona currents has been questioned, actual computation of the induced current is necessary. This necessitates knowledge of the fields produced by corona occurring near the line conductors (this will be explained in section 2). However, in literature, methods or approaches to compute the fields due to corona has not been addressed. Indeed, in a relatively recent literature, the total electric field due to a single electron avalanche has been derived and validated [3]. Since corona (and many other atmospheric discharges) is initiated and maintained by electron avalanches [2, 4], the mechanism of current induction and the propagation characteristics of the currents remain the same for avalanche and corona. Therefore, for a first study, in the present work, the analysis is carried out by considering a single avalanche developing near the surface of an overhead line conductor.

It may be added that in an isolated attempt, the corona generated current has been modelled as a series current source and the electromagnetic field has been computed by the numerical solution of Maxwell's equation (Electric Field Integral Equation or EFIE) [5]. The consequences of such a model will be briefly examined later in this work.

b) For the current computed above, the electromagnetic fields and the propagation characteristics of the currents needs to be examined. This investigation could answer the questions raised in literature on the use of transmission line model for analysis.

These form the objectives of the present work. Once the proposed approach is testified, it could be employed to quantify the noise produced by corona on overhead lines. This is to be taken up later in a separate work.

The simulation results presented is for a smooth cylindrical conductor of overall diameter $31.77 \mathrm{~mm}$, placed at a height of $10 \mathrm{~m}$ over a perfectly conducting ground. The line-to-line voltage is considered to be $400 \mathrm{kV}$.

\section{Genesis of the Induced Current}

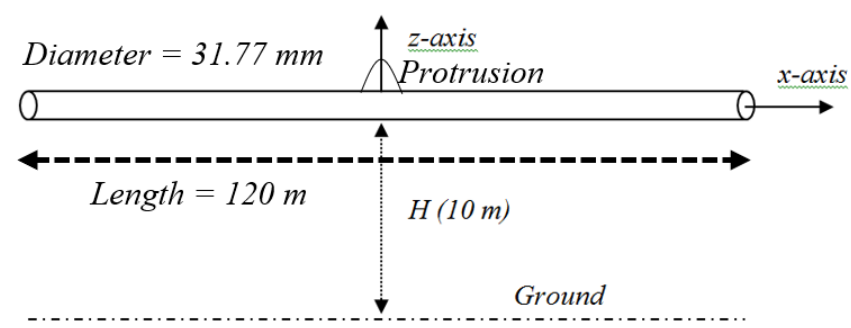

Fig.1. Conductor configuration with pertinent coordinate axes

Figure 1 shows the geometry considered, which involves a conductor above ground along with a surface irregularity/protrusion. Such surface abrasion can appear on the conductor during operation and installation. They form potential sites for the development of electron avalanches directed radially from the conductor surface (z-axis, in this case).

The dynamic electric field produced by an electron avalanche developing in air is given by equation 1 and has been derived and validated in [3]

$\vec{E}_{a v a}(\vec{r}, t)=\frac{\llbracket q \rrbracket}{4 \pi \varepsilon_{0} \llbracket \mathcal{K}^{3} \rrbracket} \llbracket \frac{1}{r^{2}}\left(\hat{r}-\frac{\vec{v}}{c}\right)\left(1-\frac{v^{2}}{c^{2}}\right)+\frac{1}{c r}(\hat{r} \times$ $\left.\left(\hat{r}-\frac{\vec{v}}{c}\right) \times \frac{\vec{a}}{c}\right) \rrbracket+\frac{1}{4 \pi \varepsilon_{0}} \llbracket \frac{\partial q}{\partial t}\left(\frac{\hat{r}}{c^{2} \mathcal{K}^{2} r} \hat{r} \cdot \vec{v}-\frac{1}{c^{2} \mathcal{K}^{2}}\left(\frac{\vec{v}}{r}\right)\right) \rrbracket+$ $\frac{1}{4 \pi \varepsilon_{0}} \int_{0}^{\llbracket R_{r} \rrbracket} \frac{\hat{r}}{r^{2}} \lambda \partial l$

Where, $q$ is the charge at the moving avalanche head, $\vec{v}$ and $\vec{a}$ are respectively the velocity and acceleration of the avalanche head, $\vec{r}$ is the vector from measurement point to source point, $r=|\vec{r}|, \hat{r}$ is unit vector along $\vec{r}, \lambda$ is the linear space charge density and $\mathcal{K}=1-\frac{\hat{r} \cdot \vec{v}}{c}$.

Clearly in equation 1 , the terms varying as $\frac{1}{r^{2}}$ and $\frac{1}{r}$ i.e., the near field and radiation terms are in separable form. Therefore, the induced currents due to these terms can be separately studied. This would be carried out in section 4.2.

When an avalanche develops near a line conductor, the electric field produced by it (given by equation 1) illuminates the conductor (termed hereafter as the incident field). As the conductor reacts to the incident field, in order to ensure zero net fields in its interior, displacement of charges takes place. In other words, the conductor produces its own electric field $\vec{E}_{\text {conductor }}=-\nabla \emptyset-\frac{\partial \vec{A}}{\partial t}$ in order to cancel the incident field due to the avalanche $\vec{E}_{\text {ava }}$.

Where $\vec{A}$ is produced by the current in the conductor and $\varnothing$ by the charge (which is related to the current by the continuity equation). This current arsing in order to cancel the effect of $\vec{E}_{a v a}$ is the genesis of the induced current in line conductors (under corona).

In order to quantify the induced current, it is necessary to evaluate $\vec{E}_{a v a}$ and therefore to trace the avalanche. The avalanche develops under the background electric field which is complicated by the presence of surface protrusions and space charges. The relevant aspects are discussed in the following.

\section{Tracing an Electron Avalanche}

\subsection{Background Electric Field Responsible for Avalanche}

Around the transmission line conductors, the avalanches develop near the surface protrusions where the electric field is locally intensified. The background electric field (under which the avalanches develop) can be thought to be the resultant of the following field components:

a). The electric field due to smooth cylindrical conductor 
b). space charge field due to previous avalanches

c). The field due to surface protrusion

The field due to smooth conductor is given by,

$$
\vec{E}_{\text {smoothConductor }}=\frac{V}{\ln \left(\frac{H}{a}\right) r} \hat{r}
$$

and has been shown in figure 2 (a) and (b) with,

$$
V=400 \times \sqrt{\frac{2}{3}} k V=326.6 k V \text { (peak value of phase }
$$
voltage for $400 \mathrm{kV}$ line-to-line.

$a=31.77 / 2 \mathrm{~mm}=15.885 \mathrm{~mm}$ is the conductor radius and $\mathrm{H}=10 \mathrm{~m}$ is the clearance from ground.

The fields due to protrusion and space charges left behind by previous avalanches involve significant uncertainty owing to the randomness in the shapes and dimensions of the protrusions. Moreover, for actual physical lines, there are no measurement or experimental verification of the space charge distribution [6]. Therefore, extensive modelling is avoided for the fields due to space charge and protrusion and simplified approaches are employed to estimate them.

The effect of space charge is modelled as described in [7]. The main features of this model are:

a). The negative charges (electrons) can be assumed to be in a sphere of radius $\sqrt{6 D t}$

where, $D$ is the diffusion coefficient, $t$ is the time from initiation of the avalanche

b). The positive ions can also be assumed in a sphere at a distance of $1 / \alpha$ [or $1 / \bar{\alpha}$ ] from the center of the sphere of the electron cloud. However, the radius of the positive ion sphere depends on the value of $1 / \alpha$ and $\sqrt{6 D t}$, whichever is higher.

Here, $\quad \bar{\alpha}=\alpha-\eta$

Where, $\alpha$ is the Townsend's first ionization coefficient and $\eta$ is the attachment coefficient [8]. Both of them and hence $\bar{\alpha}$ is a function of the ambient electric field and the number density of air $(\mathrm{E} / \mathrm{N})$. The values can be calculated from the relations available in literature [4] and given in equation 3 to 6 for reference.

$$
\begin{aligned}
& \text { For }(\mathrm{E} / \mathrm{N})>1.5 \times 10^{-15} \mathrm{~V} \mathrm{~cm}^{2} \\
& \frac{\alpha}{\mathrm{N}}=2.0 \times 10^{-16} \exp \left[\left(-7.248 \times 10^{-15}\right) /(\mathrm{E} / \mathrm{N})\right] \mathrm{cm}^{2} \\
& \text { For }(\mathrm{E} / \mathrm{N}) \leq 1.5 \times 10^{-15} \mathrm{~V} \mathrm{~cm}^{2} \\
& \frac{\alpha}{\mathrm{N}}=6.619 \times 10^{-17} \exp \left[\left(-5.593 \times 10^{-15}\right) /(\mathrm{E} / \mathrm{N})\right] \mathrm{cm}^{2} \\
& \text { For }(\mathrm{E} / \mathrm{N})>1.05 \times 10^{-15} \mathrm{~V} \mathrm{~cm}^{2} \\
& \frac{\eta}{\mathrm{N}}=8.889 \times 10^{-5}(\mathrm{E} / \mathrm{N})+2.567 \times 10^{-19} \mathrm{~cm}^{2} \\
& \text { For }(\mathrm{E} / \mathrm{N}) \leq 1.05 \times 10^{-15} \mathrm{~V} \mathrm{~cm}^{2} \\
& \frac{\eta}{\mathrm{N}}=6.089 \times 10^{-4}(\mathrm{E} / \mathrm{N})-2.893 \times 10^{-19} \mathrm{~cm}^{2}
\end{aligned}
$$

For the case of positive applied voltage, the avalanches develop into the high field region, i.e., towards the anode. The electrons are absorbed in the anode and the positive space charge remain in the area immediately next to it $[2,9,10]$.

Following the model discussed above, the negative charges have no effect since they have been absorbed. The effect of the positive space charge is represented by a sphere of radius $(1 / \alpha)$. Near the anode, since ionization stops, $\bar{\alpha}=0$, from equations (3) to (6), $\alpha$ is computed to be $7.1 \mathrm{~cm}^{-1}$. The radius of the sphere is calculated to be $1.4 \mathrm{~mm}$. The centre is at the same distance from the anode, so that the positive charges just touch the electrode surface.

The charge density for positive corona in air has been computed in [11]. The typical (spatial peak) value of $2.2 \times 10^{17} \mathrm{~m}^{-3}$ is taken for the present estimation. The field due to space charge is computed and presented in figure 2(b) along with the field due to applied voltage.

For negative applied voltage, the avalanches develop away from the cathode into the weaker field region. The positive space charge is drawn towards and absorbed into the cathode whereas the negative charges are repelled away $[2,9,10]$. Since the space charges are located near (beyond) the ionization boundary $[2,9,10]$, they have less effect on the development of new avalanches.

According to the model employed, the positive charges have no effect since they are absorbed whereas the negative charges are repelled further away from the ionization boundary. The centre of the sphere of negative charge cloud is taken to be the ionization boundary. The radius is given by $\sqrt{6 D t}$.

The value of $D$ is taken as $0.043 \mathrm{~m}^{2} / \mathrm{s}$ [9]. In order to estimate $t$, the initial mode of negative corona, namely the Trichel pulses are referred since this mode is the closest to single electron avalanche. From literature, the typical values of the tail time of the Trichel pulse currents (50\% tail) are found to lie between 50ns to 100ns [1,2]. An average value of $75 \mathrm{~ns}$ is taken in the present analysis for the duration of the pulses i.e., $t=75 n s$.

With the above values, the radius of the sphere of negative space charge cloud is calculated to be $140 \mu \mathrm{m}$. The centre of the sphere is taken to be the ionization boundary (where electric field magnitude is $26 \mathrm{kV} / \mathrm{cm}$ ) although in reality, the head of the electron cloud is located further away from the cathode. However, since the space charge has less effect as it moves further away from the cathode, this choice leads to a cautious estimate.

The electron density of negative corona has been computed in [12] and found to typically lie between $1-2.5 \times 10^{17} \mathrm{~m}^{-3}$ The study was carried out for negative corona in oxygen. However, oxygen being an electronegative gas, predominantly influences the attachment process and not the ionization. Hence, electron density value is taken to be $2.2 \times 10^{17} \mathrm{~m}^{-3}$ (same as that for positive avalanche) in the present analysis. With the above values, the field due to space charge is computed and is shown in figure 2 (a) along with field due to applied voltage. 
Finally, to estimate the field due to protrusion, the IEEE definition for the corona is referred. It defines corona as "A luminous discharge due to ionization of the air surrounding an electrode caused by a voltage gradient exceeding a certain critical value." [13]. For a luminous discharge to occur, the discharge must reach a certain critical size (number of free electrons in avalanche head due to ionization). Depending on the size of the electrode, critical avalanche size should be about $10^{5}$ to $10^{8}$ [Rather, Nasser]. In the present work, the size is chosen as $10^{8}$ and the field intensification is assumed to be sufficient for avalanches of critical size to develop.

The intensification of field due to protrusion is represented by semi-Gaussian profiles (with peak at electrode surface) and the strength is varied so that avalanches of critical size $\left(10^{8}\right)$ can develop (under the resultant field of (a), (b) and (c), section 2,1). The fields representing the intensification due to protrusion are shown in figures 2 (a) and 2 (b) for negative and positive applied voltages. The same figures also show the resultant electric fields under the action of which the avalanches develop.

With the background electric fields estimated, the next step is to trace the electron avalanches. This is discussed in the following.

\subsection{Tracing the Electron Avalanches}

The development of the avalanche is modelled according to Townsend's theory of ionization. In this model, a single electron in moving through an electric field of suitable strength, can lead to an exponential growth in the number of free electrons due to ionizing collisions. Quantitatively, a single electron will result in $e^{\int_{0}^{z} \bar{\alpha} d z}$ free electrons due to ionizing collision (in region where $\bar{\alpha}>0$ ). $\bar{\alpha}$ being the effective ionization coefficient, given by equation 2 .

The velocity of the moving electrons is also a function of the ambient electric field and the number density of air $(\mathrm{E} / \mathrm{N})$ and is given by [8]:

$v=2.1569 \times 10^{16}(\mathrm{E} / \mathrm{N})^{0.6064} \mathrm{~cm} / \mathrm{s}$

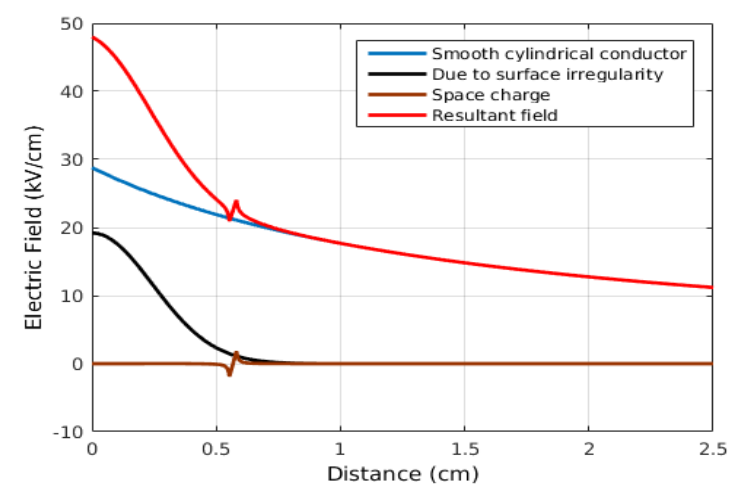

(a)

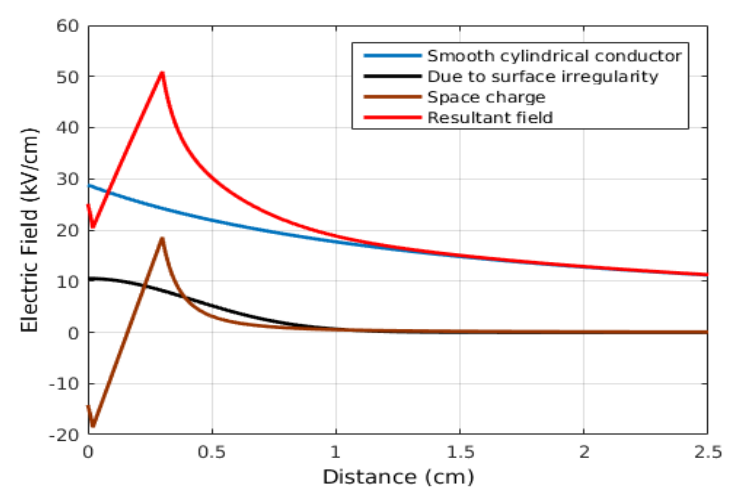

(b)

Fig.2. Background electric field profiles (a) Negative applied voltage (b) Positive applied voltage

The electron avalanches are traced according to Townsend's theory and the computed number of free electrons at the avalanche head are presented in figures 3 (a) and 3 (b).

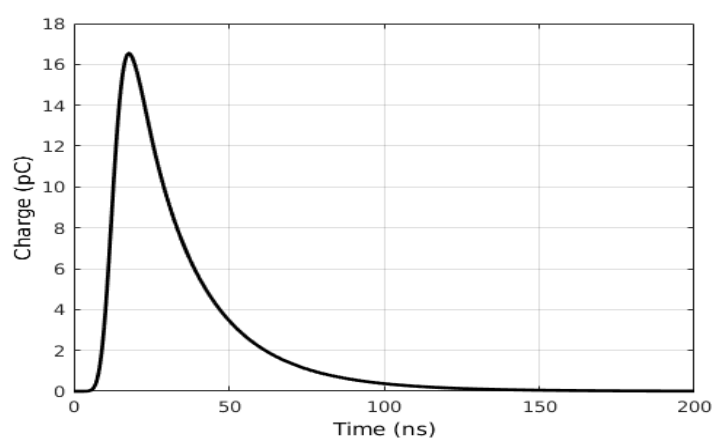

(a)

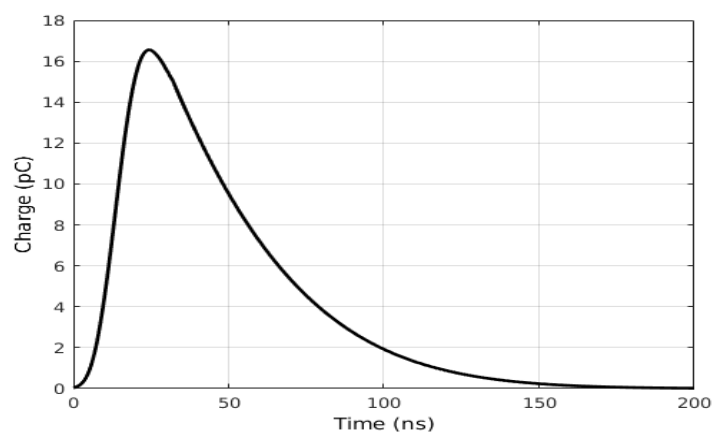

(b)

Fig. 3. Charge in the avalanche head (a) Negative avalanche (b) Positive avalanche

With the avalanches traced, the electric field produced by them can be computed from equation 1 and subsequently, the current induced by the avalanche can also be computed as will be discussed in section 4. Before proceeding, a brief discussion on the role of ground is carried out below.

\subsection{Role of Ground}

In the model for the simulation of induced current and its propagation on line conductors, a perfectly conducting ground is assumed. For the spatial length of conductor which 
is relevant for the study, this simplification is quite adequate and was verified by removing the ground altogether in the simulation for the current.

Additionally, the incident field due to the image of growing avalanche on the ground is found to be several orders lower in magnitude owing to the distance between the avalanche on the conductor and the ground (typically 8-12 m for EHV lines) and the dipolar nature of the avalanche.

In the following sections, the numerical simulation for the induced current due to a single avalanche growing near a conductor will be taken up.

\section{Numerical Computation of Induced Current due to Single Avalanche}

current

4.1 Approach to Numerically Compute the Induced

In order to compute the induced current for arbitrary line configurations and geometry, numerical computational approach needs to be adopted. In the analysis of wires, the Electric Field Integral Equation (EFIE) has been found to be suitable [14] which is followed in the present work.

Next step is to decide between time domain (TD) and frequency domain (FD) implementation. Corona currents measured and reported in literature $[1,2]$ have shown that they are short duration pulsed waveforms with a rapid rise time. For simulating phenomenon resulting in currents with above characteristics, TD methods have been found to be more suitable in literature [15]. This is therefore adopted in the present work.

Finally, for corona currents which a rise time of a few tens of $n s$, lets assume $10 n s$, the smallest wavelength of interest is $3 \mathrm{~m}$. This is about two orders of magnitude greater than the diameter of the $400 \mathrm{kV}$ line conductor $(31.77 \mathrm{~mm})$. Therefore, with suitable discretization, analysis based on TD-EFIE on thin wire structures can be implemented. This approach will satisfy the various requirements discussed above and is the method adopted in the present work.

According to the thin wire formulation [14], the electric field produced by a current filament is given by

$$
\begin{aligned}
& \vec{E}(\vec{r}, t)=-\frac{\mu_{0}}{4 \pi} \int_{C(\vec{r})}\left[\frac{\hat{s}^{\prime}}{R} \frac{\partial}{\partial t^{\prime}} I\left(s^{\prime}, t^{\prime}\right)+c \frac{\bar{R}}{R^{2}} \frac{\partial}{\partial s^{\prime}} I\left(s^{\prime}, t^{\prime}\right)-\right. \\
& \left.c^{2} \frac{\bar{R}}{R^{3}} q\left(s^{\prime}, t^{\prime}\right)\right] d s^{\prime}
\end{aligned}
$$

where, $s=s(\vec{r}), s^{\prime}=s\left(r^{\prime}\right), d s^{\prime}=d s\left(\vec{r}^{\prime}\right), R=\left|\vec{r}-\vec{r}^{\prime}\right|$ and the unprimed and primed variables denote the quantities at observation and source points respectively. $C(\vec{r})$ is the contour of the current filament carrying current $I$ [14]. $\hat{s}=$ $\hat{s}(\vec{r})$ and $\hat{s}^{\prime}=\hat{s}^{\prime}\left(\vec{r}^{\prime}\right)$ are the unit tangent vectors to $C(\vec{r})$ at $\vec{r}$ and $\vec{r}^{\prime}$.

For the incident electric field $\vec{E}_{a v a}$ produced by the avalanche on the conductor, the net tangential electric field must be zero at points on the surface of the conductor, Therefore,

$$
\hat{s} .\left(\vec{E}+\vec{E}_{a v a}\right)=0
$$

where $\vec{E}$ is evaluated at the conductor surface,

i.e. $\left|\vec{r}-\vec{r}^{\prime}\right|=a$

Therefore, using the condition from equation (9) in equation (8), $I\left(s^{\prime}, t^{\prime}\right)$ i.e., the current in the conductor is the only unknown, which for the present study is the induced current due to an electron avalanche.

To evaluate the current due to avalanche on a transmission line conductor, the conductor is discretised into segments for numerical evaluation. If $N_{s}$ is the total number of segments, then the current $I\left(s^{\prime}, t^{\prime}\right)$ can be approximated by [14],

$$
\begin{aligned}
& I\left(s^{\prime}, t^{\prime}\right) \approx \sum_{i=1}^{N_{s}} \sum_{j=1}^{\infty} I_{i j}\left(s^{\prime}-s_{i}, t^{\prime}-t_{j}\right) U\left(s^{\prime}-\right. \\
& \left.s_{i}\right) V\left(t^{\prime}-t_{j}\right)
\end{aligned}
$$

where $I_{i j}(0,0)=I_{i j}$ is the current value at the centre of the $i$ th space segment (total of $N_{S}$ ) and the $j$-th time interval (in practice a finite number $N_{T}$ ) and where

$$
\begin{aligned}
U\left(s^{\prime}-s_{i}\right) & = \begin{cases}1, & \left|s^{\prime}-s_{i}\right| \leq \Delta_{i} / 2 \\
0, & \text { otherwise }\end{cases} \\
V\left(t^{\prime}-t_{j}\right) & = \begin{cases}1, & \left|t^{\prime}-t_{j}\right| \leq \delta_{j} / 2 \\
0, & \text { otherwise }\end{cases}
\end{aligned}
$$

with $\Delta_{i}$ and $\delta_{j}$ the lengths of space segment $i$ and time interval $j$ centred at $s_{i}$ and $t_{j}$, respectively [14].

The unknown currents at the centre of each segment $I_{i j}\left(s^{\prime}-s_{i}, t^{\prime}-t_{j}\right)$ are approximated by the Lagrangian interpolation function in two dimensions [14], i.e.,

$I_{i j}\left(s^{\prime}-s_{i}, t^{\prime}-t_{j}\right)=\sum_{l=-1}^{l=1} \sum_{m=n}^{m=n+2} B_{i j}^{(l, m)} I_{i+l, j+m}$

where,

$$
B_{i j}^{(l, m)}=\prod_{p=-1}^{p=1} l \prod_{q=n}^{q=n+2} m \frac{\left(s^{\prime}-s_{i+p}\right)\left(t^{\prime}-t_{j+q}\right)}{\left(s_{i+l}-s_{i+p}\right)\left(t_{j+m}-t_{j+q}\right)}
$$

Substituting the above approximation of the unknown current in equation 8 and enforcing the condition given in equation 9 at the centre of each segment (Method of Moment or MoM formulation), the unknown current is given by the following equation [14]:

$$
\begin{aligned}
& \bar{I}_{v}=\bar{Y} \cdot\left\{\bar{E}_{v}+\sum_{l=-1}^{+1} \sum_{m=n}^{n+2} r_{i-l, u} X^{(l, m)} \bar{I}_{v-r_{i-l, u+m}}+\right. \\
& \left.\sum_{l=-1}^{+1} \sum_{r=-1}^{+1} \sum_{m=n}^{n^{\prime}+2} \sum_{t=n}^{n+2}\left\langle\frac{r_{i-l-r, u+|m|+1}}{2}\right\rangle W^{(l, m, r, t)} \sum_{s=1}^{v-r_{i-l-r, u+m}} \bar{I}_{v-r_{i-l, u+m+t-s+1}}\right\}
\end{aligned}
$$

here,

$\bar{Y}=[\bar{Z}]^{-1}$ and the elements of $\bar{Z}$ are computed from 
$Z_{u i}=\frac{\mu_{0}}{4 \pi} \hat{s}_{u} \cdot \sum_{l=-1}^{+1}\left\{\int_{-\Delta_{i-l / 2}}^{\Delta_{i-l / 2}} d s_{i-l}^{\prime \prime} \sum_{p=0}^{1} \delta\left(r_{i-l, u}-\right.\right.$

p) $\left[\frac{\hat{s}_{i-l}}{R_{i-l, u}} t B_{i-l}^{(l, p)}+c \frac{\vec{R}_{i-l, u}}{R_{i-l, u}^{2}} s B_{i-l}^{(l, p)}\right]-$

$c^{2} \sum_{r=-1}^{+1} \int_{-\Delta_{i-l-r / 2}}^{\Delta_{i-l-r / 2}} d s_{i-l-r}^{\prime \prime} \frac{\vec{R}_{i-l-r, u}}{R_{i-l-r, u}^{3}} \sum_{q=0}^{2} \delta\left(r_{i-l-r, u}-\right.$

q) $\left.B_{i-l-r}^{(l,\langle q / 2\rangle)} C_{i-r}^{\left(r,\left(\frac{q+1}{2}\right)\right)}\right\}$

and

$X_{u i}^{(l, m)}=-\frac{\mu_{0}}{4 \pi} \hat{s}_{u} \cdot\left\{\int_{-\Delta_{i-l / 2}}^{\Delta_{i-l / 2}} d s_{i-l}^{\prime \prime}\left[\frac{\hat{s}_{i-l}}{R_{i-l, u}} t B_{i-l}^{(l, m)}+\right.\right.$

$\left.\left.c \frac{\vec{R}_{i-l, u}}{R_{i-l, u}^{2}} S B_{i-l}^{(l, m)}\right]\right\}$

$W_{u i}^{(l, m, r, t)}=c^{2} \frac{\mu_{0}}{4 \pi} \hat{s}_{u} \cdot \int_{-\Delta_{i-l-r / 2}}^{\Delta_{i-l-r / 2}} d s_{i-l-r}^{\prime \prime} \frac{\vec{R}_{i-l-r, u}}{R_{i-l-r, u}^{3}} B_{i-l-r}^{(l, m)} C_{i+l}^{(r, t)}$

The electric field in the RHS of equation $12, \bar{E}_{v}=\left(\begin{array}{c}E_{1 v} \\ E_{2 v} \\ \vdots \\ E_{N_{S} v}\end{array}\right)$

Is the incident electric field at the centre of the conductor segments at the $v$ th time step. In the present study, this is computed from equation 1 at the appropriate locations and time step. Therefore, all terms in the RHS of equation 12 are known (the current terms are terms computed at an earlier time step and hence are known terms). The unknown current i.e.,

$\bar{I}_{v}=\left(\begin{array}{c}I_{1 v} \\ I_{2 v} \\ \vdots \\ I_{N_{S} v}\end{array}\right)$ at the centre of all the segments at the $v$ th time step can therefore be computed from equation 12 [14]. Repeating the procedure, the current at the centre of the segments at all time steps, i.e., computing from $v=1$ to $N_{T}$ the induced current can be computed [14].

The above numerical procedure has been implemented in an in-house code which has been testified in an earlier work [16]. For the present study, the in-house code has been used to compute the induced current due to an avalanche. The conductor is suitably discretised as discussed next.

For the numerical evaluation, length of the segments should be such that the duration of the fast-rising portion of the induced current spans at least 10 segments. Since the rise time of the current is in tens of $n s$, for $10 n s$ rise time, $0.3 \mathrm{~m}$ segment length should be sufficient. In the present work, 0.2 $\mathrm{m}$ has been taken as the segment length.

For evaluating the main portion of the current involving roughly up to $50 \%$ of the tail (about $200 \mathrm{~ns}$ ), $60 \mathrm{~m}$ of conductor length on either side of the avalanche would be adequate and hence is considered in the simulations. The dimensions considered have been specified in figure 1 .

All the essential background work being completed, in the following section, the computation of the induced current is taken up and important characteristics of the current are discussed.

\subsection{Induced Current due to an Electron Avalanche in a} Line Conductor
The induced current in the conductor shown in figure 1 due to the electron avalanches traced in figure 3 (a) and (b) are to be computed. The electric field due to the avalanches are computed from equation 1 at the centre of each conductor segment. The tangential component of the electric field is shown in figure 4 (a) and (b). The tangential field falls rapidly with distance along the conductor length, reducing by three order in magnitude by $0.5 \mathrm{~m}$ (although waveshape remains same).

Therefore, the incident tangential field is presented along the conductor length only within the first $0.5 \mathrm{~m}$ from the avalanche (on either side).

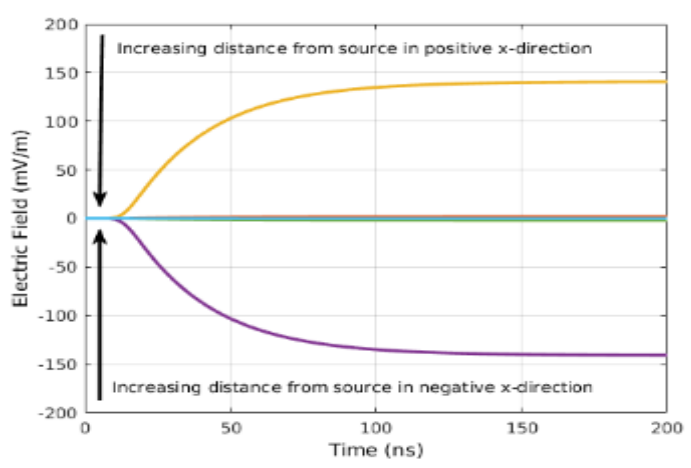

(a)

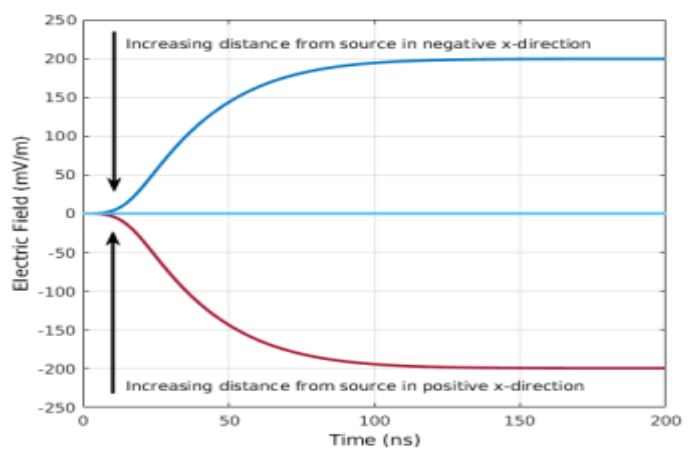

(b)

Fig.4. Temporal variation of incident tangential electric field along conductor length, within a distance of $0.5 \mathrm{~m}$ from the avalanche (a) Negative avalanche (b) Positive avalanche

The avalanche process leads to a positive charge column at its tail and negative charge region (free electrons and negative ions) at its head. Due to the geometrical proximity of one polarity of charge, the tangential field along the conductor length has only one polarity, which is directed away or towards the avalanche respectively for negative and positive avalanches.

The conductor however has been discretised into a number of segments with an associated segment vector (given by $\hat{s}=$ $\hat{s}(\vec{r}))$. In the present study, the vectors are directed along the conductor axis (Fig. 1. $x$-axis). Therefore, the field due to avalanche is directed in opposite directions on either side of it (either side of z-axis in Fig. 1). As a result, the field is bipolar in nature. With the near field dominating in the close region and the tangential field being orders of magnitude 
higher, the above description fully explain the temporal variation of the field.

With the incident field along the conductor as the input, the resulting dynamic current is evaluated using equation 12 implemented in the in-house TDEFIE code [16]. The computed current is presented in figure 6 . The current is shown at every $1 \mathrm{~m}$ along the conductor.

Similar to the incident electric field, the induced current also flows away from or towards the avalanche ( $z$-axis figure 1). Therefore, the currents and the segment vectors are directed in opposite directions on either side of the avalanche which leads to the bipolar (positive and negative) nature of the currents. For clarity, this is shown in figure 5 for induced current due to positive avalanche.

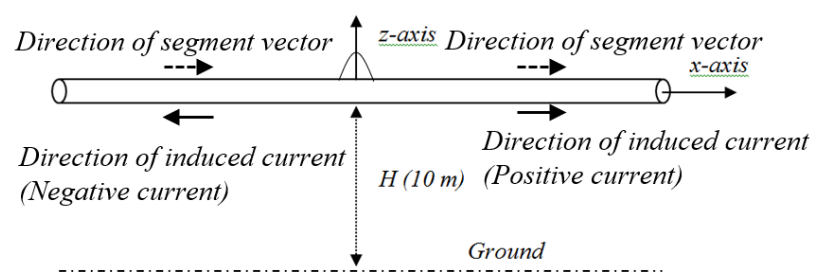

Fig. 5. Direction of segment vector and induced current in conductor due to positive avalanche

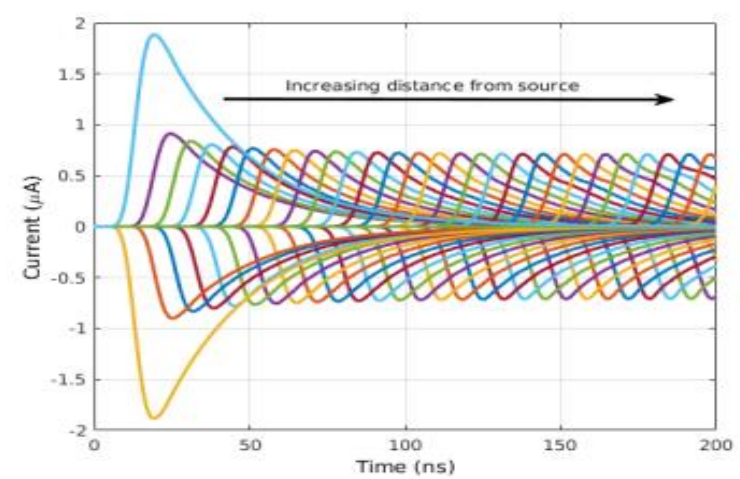

(a)

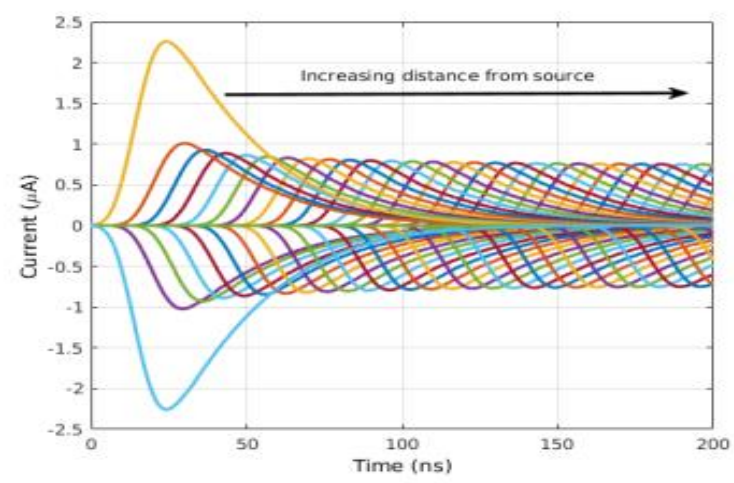

(b)

Fig. 6. Induced current in conductor due to single avalanche (a) Negative avalanche (b) Positive avalanche

As shown in figure 6 , the induced currents have a fast rise time followed by a relatively slower tail time which is typical of the measured current pulses [2]. For example, the induced current at a location of $5 \mathrm{~m}$ from the avalanche (z-axis) has a rise time of around $8 \mathrm{~ns}$ and a tail time (50\% value) of about $25 \mathrm{~ns}$ for negative avalanche. The ratio of rise time to time to tail is similar to the typical measured current pulse (Trichel pulses) [17]. The corresponding values for positive avalanche are $16 \mathrm{~ns}$ and $45 \mathrm{~ns}$ ) and the ratio of rise time to tail time is similar to typical measured current pulses (burst pulses) [18]. It is observed here that the rise time of the induced current is faster for negative avalanche than positive one. This is in line with practical corona currents measured in laboratory setups. Therefore, the results are in line with practical measurements. In the following, some of the features of the induced currents are discussed.

\subsubsection{Spatial decay in the peak value of the induced currents}

In figure 6, it is also observed that the magnitude (peak) of the induced current reduces rapidly in the initial few segments of the conductor before settling to a steady value (peak value). The decay in the peak is plotted with respect to distance from the avalanche in figure 7 . The rapid initial decay is because in the initial time regimes, the incident electric field on the conductor is cancelled by the $\frac{\partial \vec{A}}{\partial t}$ due to induced current. As temporal variation of the current decreases, the $\frac{\partial \vec{A}}{\partial t}$ term weakens necessitating the field produced by the deposited charge $(-\nabla \emptyset$ term $)$ to take over. In the region close to the avalanche having large magnitude of the incident field, corresponding large charge deposition is required. This leads to a rapid initial spatial decay in the peak of the induced current as presented in figure 7 .
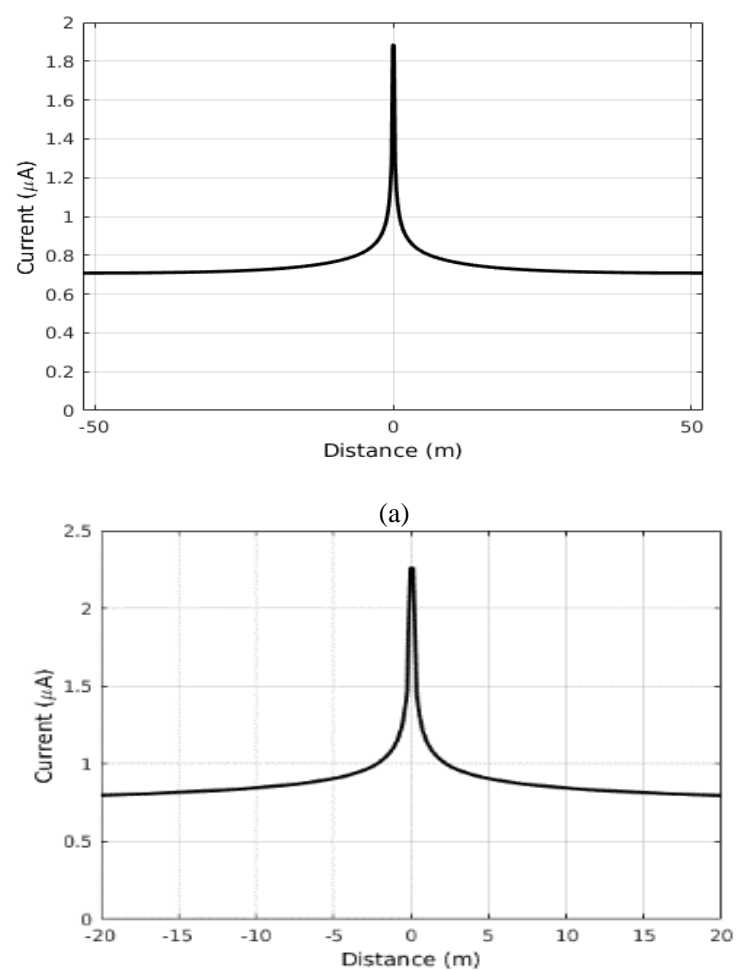

(b) 


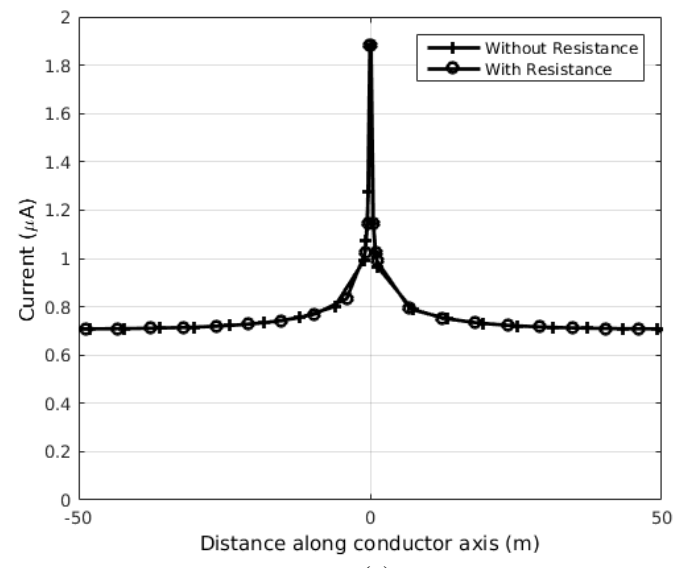

(c)

Fig.7. Magnitude of the peak of induced current along the conductor length (a) Negative avalanche (b) Positive avalanche (c) Negative avalanche with wire resistance

In order to ascertain the role played by the resistance of the line conductor, the spatial decay was recomputed by considering the resistance. The resistivity of aluminium was used and the resistance per unit length was evaluated considering the skin effect. For the induced current due to negative avalanche, with rise time of around $8 \mathrm{~ns}$, the highest significant frequency of concern is about $100 \mathrm{MHz}$ (overestimated for worst case study). The resistance, considering skin depth at this frequency was computed to be $0.0323 \Omega / \mathrm{m}$. With this resistance per unit length distributed across the entire length of the line conductor, the induced current was recomputed and the spatial decay obtained is shown in figure 7 (c). It is observed that the resistance has no effect on the spatial decay in the peak of the induced current. For current due to positive avalanche which has a slower rise time, the effect of resistance is even lower. Therefore, the rapid initial spatial decay of the induced current is due to the charge deposition process and is not influenced by the conductor resistance.

\subsubsection{Polarity of charge deposited and comparison with series current source modelling}

As has been stated earlier, corona current has been modelled as series sources in an isolated work in literature [5]. This leads to an incorrect representation of the physical phenomenon as discussed next.

The densities of the charges deposited across the source (avalanche at z-axis) by the induced current for the present study as well as the series current model [5] are presented in figure 8 .

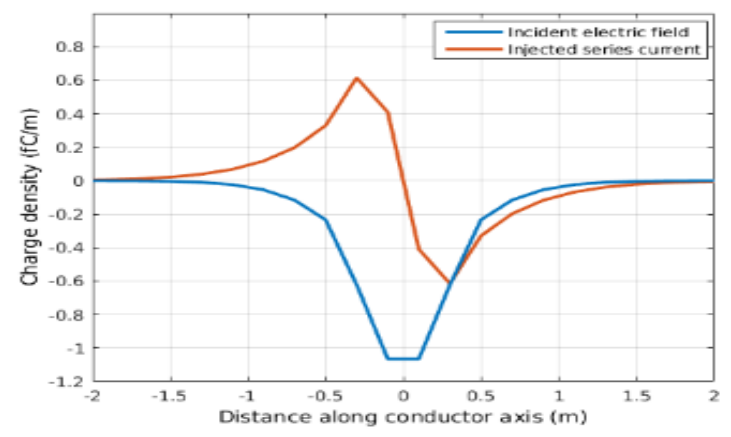

(a)

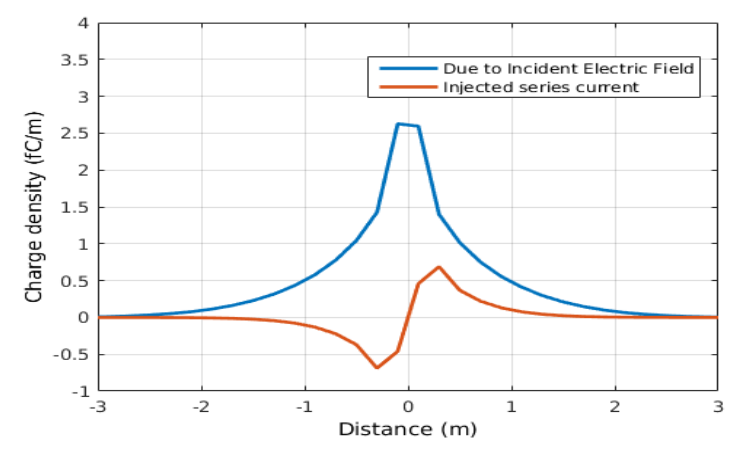

(b)

Fig.8. Charge density across source for single avalanche (a) Negative avalanche (b) Positive avalanche

Clearly, the polarity of charge deposited in the former case is unipolar, while in the latter it is bipolar. This is due to the fact that a series current source demands opposite polarity charges to flow in opposite directions which in the present case is not meaningful as corona sends equal current in either direction. It is therefore clear that even if full field-based approach is employed, modelling of corona induced current as a series current source [5] is not appropriate.

\subsubsection{Induced Current due to near field and radiation field components}

The induced current due to near and radiation components are separately studied. This is possible since the (incident) field due to an avalanche given by equation 1 is separable into the respective components. Therefore, in the RHS of equation 12 , the individual components are considered and the currents are computed and presented in figure 9 (a) and (b). Results are shown for the case of negative avalanche. However, the inference is true for positive avalanche (obviously since it possesses slower rise time) as well.
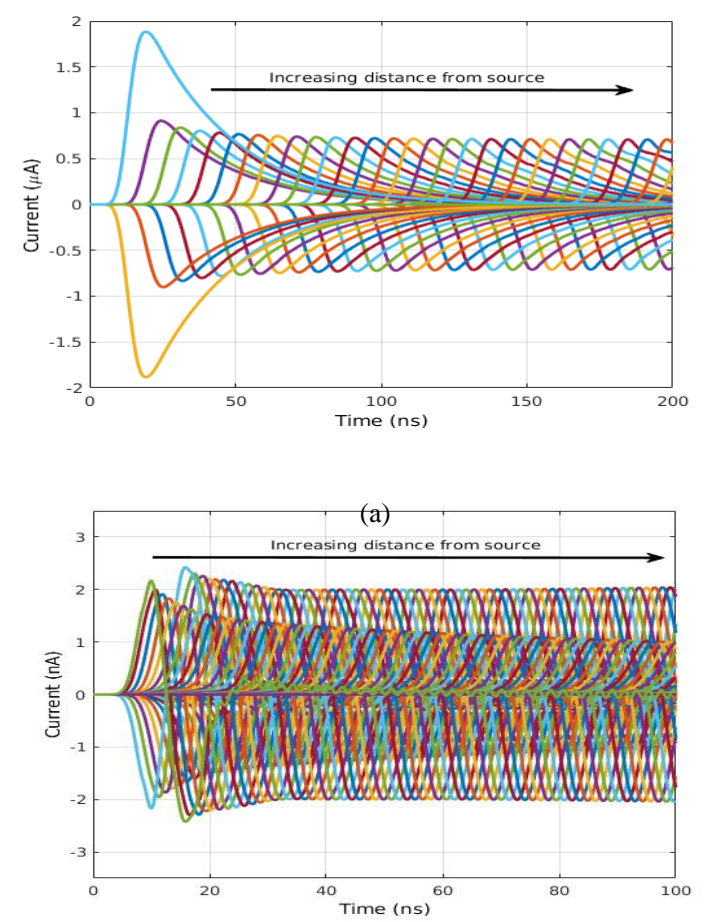

(b)

Fig.9. Induced Current due to incident field of Negative avalanche (a) Near field component (b) Radiation component 
It is observed that the current due to the static or near field completely dominates over the current due to radiation field terms by three orders of magnitude. The reason is that; since the avalanche develops close to the conductors, the near field terms of the field due to avalanche dominate over the radiation terms as has been demonstrated in literature [3].

The important inferences about the induced currents can be summarized as:

1. The currents have fast rise time followed by a relatively long tail. The rise times are faster for negative avalanche induced currents than positive avalanche.

2. The currents have a very rapid initial spatial decay which is a property of the induction process itself and shows no dependence on the conductor resistance.

3. The induced current deposits unipolar charge across the source (avalanche) on either side which is incorrect to be modelled by series current sources.

4. The current induced by the near field term dominates completely over the induced current due to radiation term.

Inferences drawn in this section were tested for different field intensification due to protrusion as well as avalanches initiated at different points between the conductor and ionization boundary. It was verified that the inferences drawn remains unchanged.

With the induced currents computed in single conductor configurations, and important inferences with regards to their basic characteristics drawn, a practical 4 conductor configuration comprising of 3-phase conductors and one ground wire is taken up for study in the following.

\subsection{Induced Current in 4 Conductor Configuration}

In this section, the current produced by a single electron avalanche (for both negative and positive avalanches) in 4conductor configuration is evaluated. The conductor configuration is shown in figure 10 .

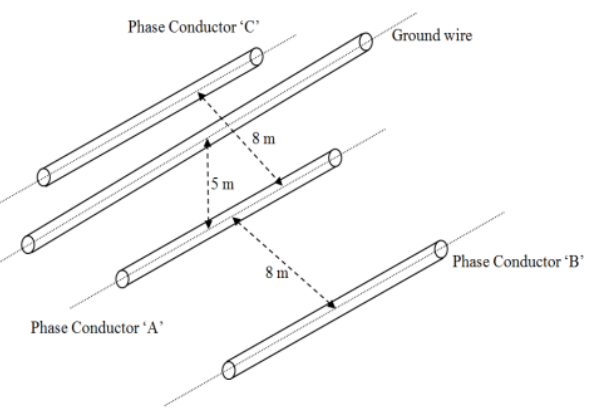

Fig.10. Four-conductor configuration

A single avalanche (of size $10^{8}$ ) is considered to develop close to the surface of the phase conductor ' $A$ ' with the other conductors free from avalanche activity. The procedure is identical to that adopted for the single conductor case, namely, the avalanche is traced and the incident electric field on all the four conductors is evaluated. The induced current is then computed with the in-house time domain numerical code.

The incident electric field in the phase conductor ' $A$ ' is identical to that obtained previously (figure 4 ). For other conductors, while the waveshape remained unaltered, the magnitude was lower by more than four orders. Therefore, the magnitude of the electric field incident on the other conductors is rather weak. The corresponding computed current in the conductors is presented in the following figures.

The induced current in the phase conductor ' $A$ ' is identical to that obtained for the case of a single conductor i.e. figure 6 . The induced current in the remaining phase conductors (B and C) are identical and is as presented in figure 11.

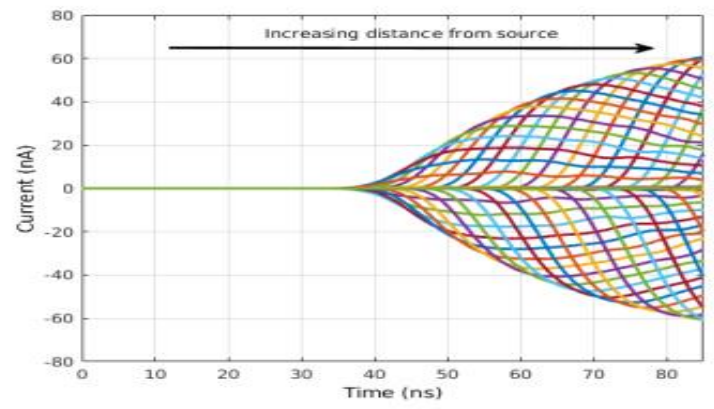

(a)

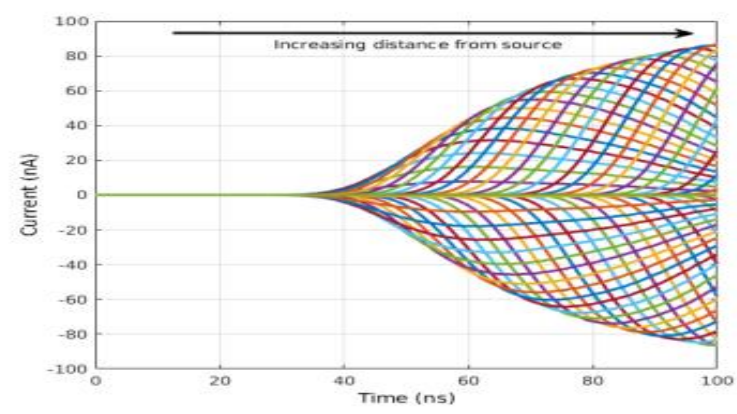

(b)

Fig.11. Temporal variation of induced current in phase conductors ' $B$ ' and ' $C$ ', presented every $1 \mathrm{~m}$ along conductor length (a) Negative avalanche (b) Positive avalanche

The current in the ground wire is shown in figure 12.

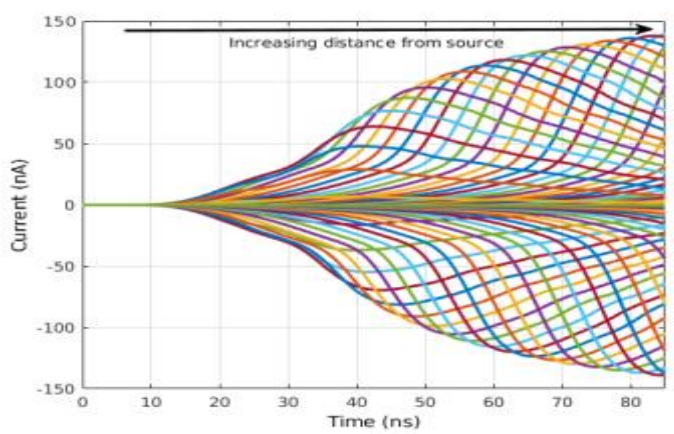

(a)

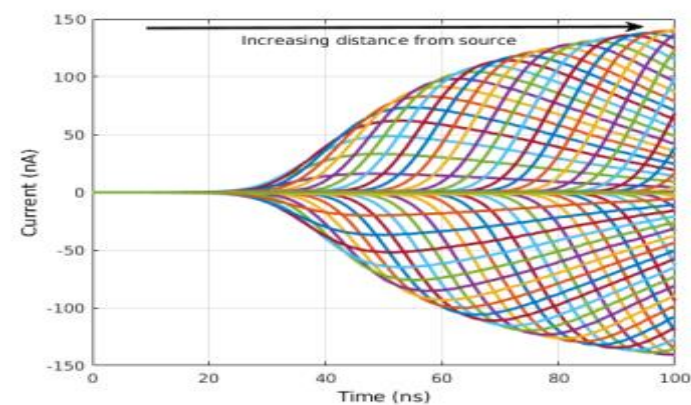

(b)

Fig.12. Temporal variation of induced current in the ground wire presented every $1 \mathrm{~m}$ along conductor length (a) Negative avalanche (b) Positive avalanche 
Even though the incident electric field in phase conductors ' $\mathrm{B}$ ' and ' $\mathrm{C}$ ' and the ground wire is at least 4 orders of magnitude lower than the field in phase conductor ' $A$ ', the current in them are only two orders of magnitude lower. This implies that the currents produced in the phase conductors ' $\mathrm{B}$ ' and ' $C$ ' and the ground wire due to the current in phase conductor ' $\mathrm{A}$ ' dominates over the current produced in them by the incident electric field.

With the induced currents in the line conductors computed and important inferences drawn, the next objective of the present work (question on the applicability of transmission line model for analysis) is addressed. This can be carried out by investigating the pattern of the electric and magnetic fields produced by the induced currents around transmission line conductors. Since the currents produced in neighbouring conductors are at least two orders of magnitude lower, only a single conductor close to which the avalanche develops is considered.

\section{Electric and magnetic fields due to induced current}

\subsection{Electric and Magnetic Fields in Long Transmission Line Model}

The long transmission line model requires the following two conditions to be satisfied with respect to the associated fields:

1) For a two-wire transmission line (or line and ground), pattern of the electric field between the wires (or wire and ground) must as shown in figure 13 (for the case of positive applied voltage) [19].

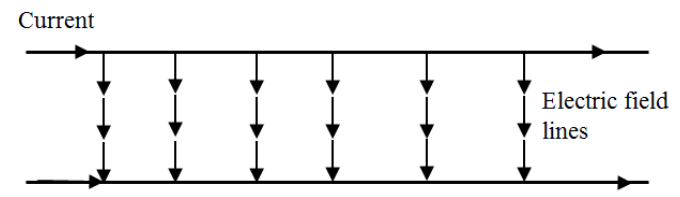

Fig.13. Electric field lines between two wire transmission lines during TEM mode of propagation

2) The magnetic field lines must be orthogonal to the electric field lines.

This pattern of electric and magnetic fields satisfying the above two conditions corresponds to the Transverse Electro Magnetic (TEM) mode of propagation which is therefore inherently assumed in applying the transmission line model.

Considering the computed induced currents due to single avalanches, the electric field at any location can be computed by solving equation 8 with $I\left(s^{\prime}, t^{\prime}\right)$ being the induced current due to single avalanche (computed already in section 4.2). Computation is carried out with the in-house code [16] (TDEFIE).

The magnetic field is computed from the electric field by applying Faraday's law as below.

$$
\nabla \times \vec{E}=-\frac{\partial \vec{B}}{\partial t} \text { or, } \vec{B}=-\int_{0}^{t}(\nabla \times \vec{E}) \partial t
$$

The evaluation of the fields is carried out at a number of grid points between conductor and ground as shown in figure 14. Also shown are the coordinate axes (referred to figure 1) whose origin is at $(0 \mathrm{~m}, 10 \mathrm{~m})$.

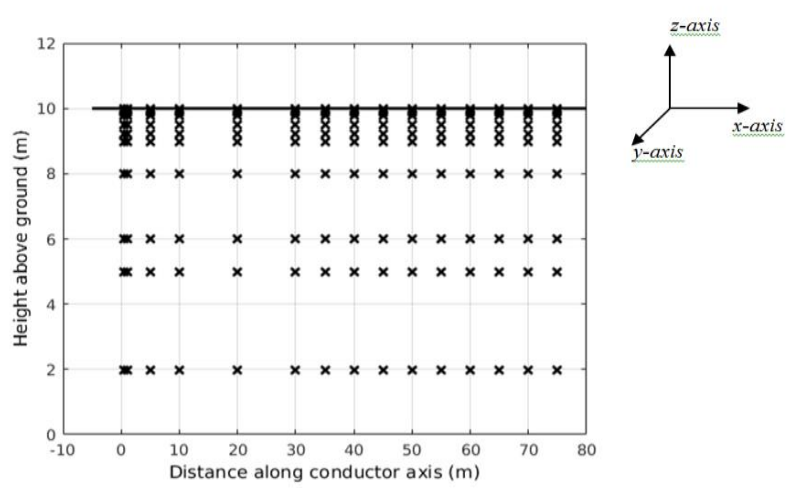

Fig.14. Measurement points between conductor and ground to study propagation of the fields

The directions of the computed fields are presented in figures 15 and 16 respectively for induced currents due to negative and positive avalanches. It is worthwhile to mention that similar to the depiction in figure 13, the arrows denote only the direction of the fields. The length of the arrows does not have any relation to the magnitude of the field which obviously diminish with distance.

It is found that the magnetic field is perpendicular to the direction of the electric field at all points and for all time. Referring to figure 14 the magnetic field had only non-zero $y$-component (shown by 'cross' in figure 15 and dot in figure 16). The $x$ and $z$ components were identically zero at all-time instants.

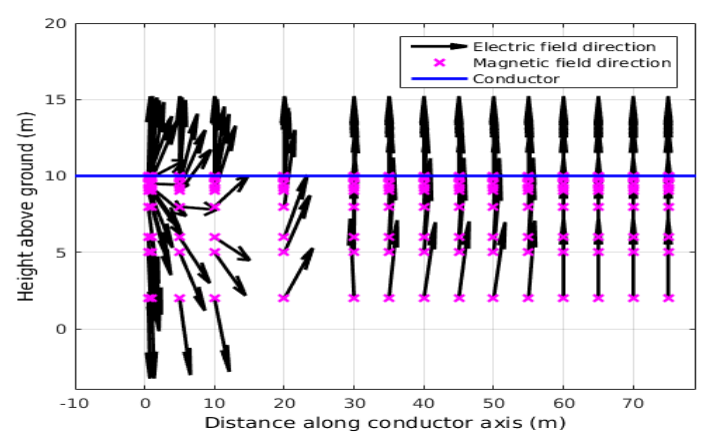

Fig.15. Direction of electric and magnetic fields due to induced current in the conductor for negative avalanche

On the other hand, it is observed that as the current propagates along the length of the conductor, initially, the electric field (produced by the induced current), is not orthogonal to the conductor axis i.e., a component of the electric field exists parallel to the axis.

Only beyond a certain distance $(60 \mathrm{~m}$ for negative and 50 $\mathrm{m}$ for positive avalanche) from the source, the electric field lines resemble the pattern in figure 13. This implies that it is only beyond this distance, that the pattern of electric and magnetic field corresponds to that of classical TEM mode of propagation and consequently, the transmission line model becomes applicable. 


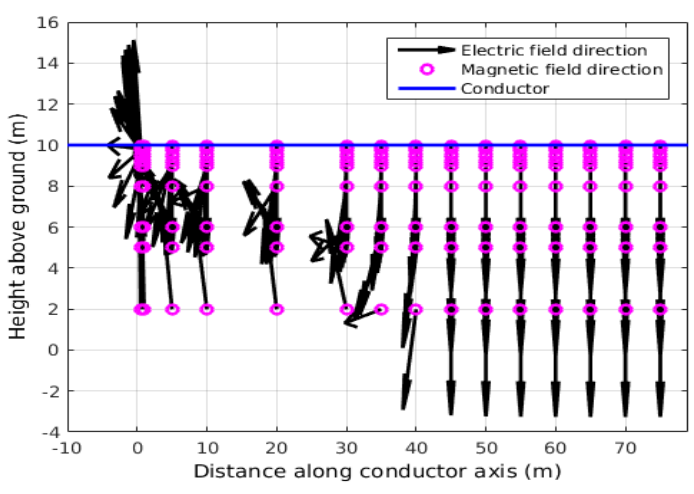

Fig.16. Direction of electric and magnetic fields due to induced current in the conductor for positive avalanche

It is recalled that the rise time of the computed induced current due to negative avalanche is $8 \mathrm{~ns}$ and that due to positive avalanche is $16 \mathrm{~ns}$. The analysis was repeated for current waveforms having different rise times and tail times for the same conductor geometry and line configuration. It was observed from the simulations that with the conductor diameter and the line configuration unchanged, the faster the rise time of the current pulse, the longer was the distance from the source at which TEM mode of propagation can be realized.

By artificially slowing down the avalanche such that the induced currents had rise times similar to measured corona current (10 ns and $50 \mathrm{~ns}$ for negative and positive corona currents respectively), the field patters were computed again. The distance beyond which the classical TEM mode of propagation could be realized was found to be close to the previous values i.e., $60 \mathrm{~m}$ and $45 \mathrm{~m}$ respectively. Although the exact distance depends on the rise time and possibly on the line geometry and configuration, it is evident that classical TEM mode cannot accurately represent the fields for a few tens of metres from the avalanche location.

In practice, under fair weather conditions, corona sources are separated by a distance of only 20-50 $\mathrm{cm}$ [5]. Therefore, from the above analysis, it is clear that well before the electric fields can assume the pattern shown in figure 13, a new corona source is encountered. Therefore, classical TEM mode of propagation is an assumption which is not strictly valid in practical scenario. As a result, applicability of transmission line model has been rightfully questioned in literature and analysis based on the same may lead to erroneous results.

\section{Conclusion}

The present work adopted a complete theoretical approach (based on Maxwell's equation) to study the currents induced by the basic discharge process of electron avalanche. The avalanche was modelled by the well-known Townsend's ionization process and the electric field due to the same was computed using an expression derived and validated in literature. The effect of the electric field of an avalanche when incident on a line conductor is studied and the genesis of the induced current is studied. This is followed by numerical computation of the induced current. The waveshapes of the computed currents are found to agree well with the measured currents reported in the literature. Some of the important characteristics of the induced currents are studied. From the nature of the charge deposited along the conductor, it is shown that a series current source modelling of corona current carried out in literature does not correctly represent the actual mechanism.

The induced current in a practical 4-wire configuration is studied. Finally, the field due to avalanche/corona current induced in the line conductor was evaluated and it is shown that up to a few tens of meters from the source, the classical transmission line model is not very appropriate. Questions raised in literature regarding the use of transmission line models have been found to be quite appropriate and the reason behind the same has been demonstrated.

\section{References}

[1] J.J. LaForest, "Transmission-line reference book. $345 \mathrm{kV}$ and above", General Electric Co., Pittsfield, MA (USA). Large Transformer Div.; General Electric Co., Schenectady, NY (USA). Electric Utility Systems Engineering Dept., 1981.

[2] P.S. Maruvada, "Corona performance of High-Voltage Transmission Lines", Baldock, UK, Research Studies Press, 2000.

[3] D. Nath, U. Kumar, "Total electric field due to an isolated electron avalanche," IEEE Transactions on Dielectrics and Electrical Insulations, 23, no 5, pp. 2562-2571, 2016.

[4] V. Cooray and G. Cooray, "Electromagnetic radiation field of an electron avalanche", Atmospheric research, 117, pp.18-27. 2012

[5] S.K. Nayak, M. J. Thomas, "An integro-differential equation technique for the computation of radiated EMI due to corona on HV power transmission lines", IEEE transactions on power delivery, 20, no 1, pp. 488-493, 2005

[6] H. Liu, R. Liao, X. Zhao, and Y. Lin, "The effect of air pressure on the surface electric field intensity characteristics under negative DC corona discharge in a corona cage", International Journal of Electrical Power \& Energy Systems, 113, pp.244-250, 2019.

[7] H. Parekh, K. D. Srivastava, "Effect of avalanche space charge field on the calculation of corona onset voltage," IEEE Transactions on Electrical Insulation, 4, pp. 181-192, 1979.

[8] L.B. Loeb, Electrical Coronas: their Basic Physical Mechanisms, University of California press, 1965

[9] E. Nasser, "Fundamentals of gaseous ionization and plasma electronics", Wiley-Interscience, 1971.

[10] J. Kuffel, P. Kuffel, "High voltage engineering fundamentals", Elsevier, 2000

[11] R. Morrow, "The theory of positive glow corona," Journal of Physics D: Applied Physics, Vol. 30, No. 22, pp. 3099, 1997

[12] R. Morrow, "Theory of negative corona in oxygen," Physical Review A,32(3), pp. 1799, 1985

[13] IEEE Standard Definitions of Terms Relating to Corona and Field Effects of Overhead Power Lines, IEEE Std 539-2005 (Revision of IEEE Std 539- 1990), 2005

[14] E.K. Miller, A.J. Poggio, G.J. Burke, “An integro-differential equation technique for the time domain analysis of thin wire structures. I. The numerical method," Journal of Computational Physics, Vol. 12, No. 1, pp. 24-48, 1973

[15] S.M. Rao, ed., Time domain electromagnetics. Elsevier, 1999.

[16] R.K. Govinda, "Lightning Threat to Cables on Tall Towers and the Question of Electrical Isolation," Ph.D. Thesis, Department of Electrical Engineering, Indian Institute of Science, India, 2014

[17] K. Shang, X. Xue, X. Wang, "Trichel Pulse Characteristics in Negative dc Corona Discharge." Power and Energy Engineering Conference (APPEEC), Asia-Pacific. IEEE, 2011

[18] N.T. Giao, J.B. Jordan, "Modes of corona discharges in air," IEEE Transactions on Power Apparatus and Systems 5, pp. 1207-1215, 1968.

[19] E.C. Jordan, K. G. Balmain, "Electromagnetic Waves \& Radiating Systems", New Delhi, India, Prentice-Hall of India Private limited, 2006 